\title{
Análisis clínico de 75 casos de sepsis en el lactante del Servicio de Pediatría del Hospital Regional Leonardo Guzmán de Antofagasta
}

Dres. Manuel Zamorano G.*, Manuel Yáñez Z.* y Paulino Guerra A.**

Las enfermedades infecciosas resultan de la interacción del poder agresor de un germen, en un huésped con capacidad de resistencia limitada, en un determinado medio ambiente ${ }^{43.44-47}$ El período de lactante constituye una etapa de mayor riesgo derivado de: 1 . Rápido crecimiento y desarrollo; 2 . Inmunidad reducida; 3. Deficientes condiciones ambientales de nuestro medio que impactan más fácilmente en el período de la vida señalado, y 4 . Rapidez y frecuencia con que distintas enfermedades se agravan $y$ requieren hospitalización y el empleo de técnicas (fleboclisis, croupettes, etc.) y tratamientos que facilitan el contagio ${ }^{13-14.2122-}$ 25-43.44. Por otro lado, la mejor administración de los Servicios de Pediatría para cubrir la siempre creciente demanda de atención lleva a un mayor movimiento, especialmente en las salas de lactantes. Si a lo anterior sumamos el empleo indiscriminado e inadecuado de antibióticos, se consigue por un lado seleccionar cepas resistentes, y por otro lado se está facilitando su difusión $22.23-24.2538-43-45-46$. Es así como los gérmenes habituales condicionantes de

*Depto. Ciencias de la Salud, Universidad de Chile, Sede Antofagasta.

* Servicio de Pediatría. Hospital Leonardo Guzmán de Antofagasta. cuadros bien conocidos por los clínicos han sido reemplazados por otros como Pseudomonas, aerobacter aerogenes, Klebiella aerobacter, serratia marcesens, citrobacter, anaerobios, hongos y virus, por destacar algunos que condicionan patología severa, a veces poco conocida y de dificil tratamiento 1 2-3-4-5-6-7-8-9-10-11-12-13-14-

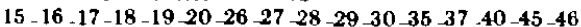

El éxito en el tratamiento de una patología infecciosa depende, por un lado, del terreno afectado y, por otro, de las medidas que se aplican, y en esto tienen gran importancia la oportunidad del tratamiento y luego la efectividad del mismo. En este sentido los antibióticos han significado un a ayuda inestimable, pero en su utilización debe considerarse la susceptibilidad del germen, la vía de introducción, la toxicidad del medicamento, la farmacodinamia del mismo y además su costo $31-32-33-34-36-38-40-42$

A medida que los gérmenes habituales disminuyen su virulencia o por lo menos la frecuencia y gravedad de los cuadros que desencadenan, hemos de preocuparnos cada vez más de estas nuevas temibles amenazas a nuestros enfermos. Es así como estamos plenamente convencidos que un porcentaje no despreciable de sepsis clínica sin comprobación bacteriológica en nuestros laboratorios corresponde a infecciones por anaerobios, virus y/o hongos ${ }^{26 \_27-28 \cdot 29-30}$. 


\section{MATERIAL Y METODO}

Se analizan 75 casos de lactantes hospitalizados en el Servicio de Pediatría del Hospital Leonardo Guzmán de Antofagasta, desde marzo de 1973 a febrero de 1978. Se incluyen los pacientes con cuadro clínico sugerente y comprobación bacteriológica. Casi la totalidad de los casos tiene uno o más hemocultivos positivos. Se incluyen seis casos sin hemocultivo, pero con hallazgo del agente patógeno en muestras de pus de abscesos, articulaciones u otra localización confiable y que además tenían un cuadro clínico concordante. Se excluyen aquellos pacientes en que no se logró identificación del germen. Tampoco se incluyen los pacientes con salmonellosis, que fueron objeto de otro estudio.

Se realizaron los siguientes estudios:

- Hallazgos bacteriológicos.
- Distribución por edad, sexo y estado nutritivo.

- Incidencia estacional.

- Días de hospitalización.

- Diagnóstico de ingreso.

- Cuadro clínico.

- Características de hemograma.

- Tratamiento antibiótico.

- Letalidad según etiología, edad y estado nutritivo.

\section{RESULTADOS}

\section{Hallazgos BaCTERIOLÓgicos}

De los 75 casos estudiados, 69 tenían hemocultivos positivos. En 6 casos el germen se aisló del LCR, pus de abscesos, herida de denudación y en un caso en orina. La distribución se analiza en la Tabla 1.

En 34 casos se aisló enterobacter sp., lo que

Tabla 1

DISTRIBUCION SEGUN FRECUENCIA DE LOS GERMENES ENCONTRADOS 75 LACTANTES CON SEPTICEMIA

\begin{tabular}{lcc}
\hline Germen & $N^{\infty}$ cosos & $\%$ \\
\hline Enterobacter sp. & 34 & 45,4 \\
Pseudomonas & 12 & 16,0 \\
KJebsiella & 7 & 9,3 \\
Estafilococo coag. ( +) & 4 & 5,3 \\
Estafilococa coag. ( - & 4 & 5,3 \\
E. coli & 3 & 4,0 \\
Gram (-) no fermentador & 2 & 2,7 \\
Proteus & 2 & 2,7 \\
Citrobacter & 2 & 2,7 \\
Alcaligenes sp. & 2 & 2,7 \\
Enterococo & 1 & 1,3 \\
Neumococo & 1 & 1,3 \\
Hemophillus influenzae & 1 & 1,3 \\
\hline Total & 75 & 100,0 \\
\hline
\end{tabular}

constituye el $45,4 \%$, le sigue en frecuencia la pseudomona con 12 casos y el $16 \%$. La Klebsiella se aisló en 7 casos con 9,3\%. El estafilococo, sumando los coagulosa positivos y los negativos, alcanzó a 8 casos con $10,6 \%$. En total los gérmenes Gram (-) alcanzaron un $86,8 \%$.
En la Tabla 2 se analizan 6 casos incluidos aunque no tenían hemocultivo. En un caso el germen se aisló simultáneamente de dos muestras. En los restantes el cuadro clínico era muy severo y el germen fue aislado de muestras confiables. 
Tabla 2

ORIGEN MUESTRA Y GERMEN AISLADO EN 6 CASOS SIN HEMOCULTIVO

\begin{tabular}{lll}
\hline \multicolumn{1}{c}{ Muestra } & \multicolumn{1}{c}{ Resultados } \\
\hline Orina y pus absceso & 1 caso & (Pseudomona) \\
Pus abscesos & 2 casos & (Pseudomona y Neumococo). \\
Pus herida denudación & 1 caso & (Pseudomona). \\
LCR & 1 caso & (Hemophillus influenzae). \\
Orina P. vesical & 1 caso & (E. Coli). \\
\hline Total & 6 casos \\
\hline
\end{tabular}

DistRIBUCión POR EDAD, SEXO Y ESTADO NUTRITIVo (Tabla 3)

Treinta y nueve casos se presentaron bajo los 6 meses. 25 casos entre los 6 y 11 meses. Se comprueba así una mayor frecuencia entre los lactantes de menos edad, alcanzando a $85,3 \%$ bajo el año de edad.

Otro aspecto de interés es que 53 pacientes, $70,7 \%$, eran desnutridos, ratificando el peor te- rreno que representan estos casos. También se destaca el predominio del sexo masculino, que se comprometió en 50 casos $(67 \%)$ contra 25 del sexo femenino (33\%).

INGIDENCIA ESTACIONAL

Como se analiza en la Tabla 4, la mayor frecuencia se aprecia durante el verano, con $39 \%$ de los

Tabla 3

DISTRIBUCION DE 75 LACTANTES CON SEPTICEMIA DE ACUERDO A EDAD, ESTADO NUTRITIVO Y SEXO

\begin{tabular}{|c|c|c|c|c|c|c|}
\hline \multirow[b]{2}{*}{ Grupo etario } & \multicolumn{2}{|c|}{ Sexo } & \multicolumn{2}{|c|}{ Est. nutrit. } & \multirow[b]{2}{*}{$N^{\infty}$ de casos } & \multirow[b]{2}{*}{$\%$} \\
\hline & 8 & 9 & Eutrof. & Desnut. & & \\
\hline 28 días -5 meses & 26 & 13 & 14 & 25 & 39 & 52,0 \\
\hline $6-11$ meses & 18 & 7 & 7 & 18 & 25 & 33,3 \\
\hline $12-17$ meses & 5 & 3 & 0 & 8 & 8 & 10,7 \\
\hline $18-23$ meses & 1 & 2 & 1 & 2 & 3 & 4,0 \\
\hline Total & 50 & 25 & 22 & 53 & 75 & 100,0 \\
\hline
\end{tabular}

Tabla 4

DISTRIBUCION DE 75 CASOS DE SEPTICEMIA DEL LACTANTE DE ACUERDO A LA INCIDENCIA ESTACIONAL Y POR ANNO

\begin{tabular}{lrrrrrrrr}
\hline \multirow{2}{*}{ Estación } & \multicolumn{9}{c}{$A N$ O } & Total & $\%$ \\
\cline { 2 - 8 } & 73 & 74 & 75 & 76 & 77 & 78 & & \\
\hline Verano & - & 2 & 4 & 7 & 21 & 5 & 39 & 52,0 \\
Otoño & 1 & - & 4 & 4 & 1 & - & 10 & 13,3 \\
Invierno & - & - & 1 & 2 & 7 & - & 10 & 13,3 \\
Primavera & - & 1 & - & 9 & 6 & - & 16 & 21,4 \\
\hline Total casos & 1 & 3 & 9 & 22 & 35 & 5 & 75 & 100,0 \\
\hline
\end{tabular}


casos. El porcentaje restante se distribuye con frecuencia casi homogénea en las otras estaciones del año. Esta mayor frecuencia en verano puede explicarse por el hecho de que la patología previa más frecuente fue el síndrome diarreico agudo y el hecho de que en este período el sector de lactantes se recarga de enfermos alcanzando incluso un índice ocupacional superior al $100 \%$. Esto, que evidentemente significa el agregado de camas supernumerarias, explica, por un lado, una mayor frecuencia de fleboclisis y otras técnicas de enfermería y, por otro lado, una menor relación auxiliar/cama, lo que, sin duda, incide en la eficiencia de la atención y en la mayor posibilidad de contagio intrahospitalario.

\section{DIAS DE hOSPITALIZACIÓN Y DIAGNÓSTICO DE INGRESO}

El promedio de días de estada fue de 30 (rango de 6 a 76 días). En cuanto al diagnóstico de ingreso, se analiza en la Tabla 5, consignando el que motivó su hospitalización y el que revestía mayor trascendencia inmediata para la vida del niño.

\section{Tabla 5}

DIAGNOSTICO DE INGRESO DE 75 LACTANTES CON SEPTICEMIA

\begin{tabular}{lcc}
\hline Diagnóstico & $N^{\circ}$ casos & $\%$ \\
\hline Síndrome diarreico aguda con deshidratación & 51 & 68,0 \\
Infecciones respiratorias agudas & 9 & 12,0 \\
Sepsis & 5 & 6,7 \\
Sindrome febril en estudio & 5 & $\mathbf{6 , 7}$ \\
Otros & 5 & $\mathbf{6 , 6}$ \\
\hline Total & 75 & $\mathbf{1 0 0 , 0}$ \\
\hline
\end{tabular}

Como se aprecia en la Tabla 5, en 51 casos el diagnóstico de ingreso fue de síndrome diarreico agudo con deshidratación, patología que constituye el proceso de base más frecuente. Le sigue en orden de frecuencia las infecciones respiratorias agudas (Bronconeumonías y Bronquitis obstructivas agudas) con 9 casos $(12 \%)$. El resto de las enfermedades tienen una distribución similar de 5 casos $(6,7 \%)$.

Hallazgos semiológicos. Son analizados en el gráfico 1 en orden de frecuencia decreciente en que se encontraron.

Recuento Leucocitario. En esta ocasión sólo analizaremos el recuento de leucocitos, siguiendo el criterio de Derman para estimar los casos como cifra normal, disminuida o aumentada. (Tabla 6). Sólo se consideraron 72 casos, ya que en 3 este examen no se consignó en la historia clínica. Se aprecia que el $55,5 \%$ evolucionó con cifra de leucocitos dentro de lo normal. El $37,5 \%$ lo hizo con leucocitosis y el $7 \%$ restante con leucopenia.
Tabla 6

RECUENTO DE LEUCOCITOS EN 72 CASOS DE LACTANTES CON SEPSIS

\begin{tabular}{lcc}
\hline$N^{\circ}$ de leucocitos & $N^{\circ}$ casos & $\%$ \\
\hline-5.000 & 5 & 7,0 \\
$5.000-18.000$ & 40 & 55,5 \\
$18.001-0$ más & 27 & 37,5 \\
\hline Total & 72 & 100,0 \\
\hline
\end{tabular}

Evolución. El promedio de dias previos a la enfermedad fue de 6 , la fiebre tuvo un promedio de duración de 12 días, la deshidratación de 6 y la alteración de las deposiciones alcanzó a un promedio de 6 días. En 73 pacientes debió emplearse hidratación parenteral por fleboclisis con un promedio de 8 días de mantención.

Letalidad. De acuerdo a la Tabla 7, de 75 casos, fallecieron 13, lo que corresponde a un $17,2 \%$; el mayor número de casos fatales se observó en el menor de un año, lo que está en relación con el mayor número de casos en este período. 
GRAFICO 1

SINTOMAS Y SIGNOS SEGUN PRECUENCLA EN 75 LACTANTES CON SEPTIC EMIA

Decaimiento
Diarrea
Fiebre
Palidez
Deshidratación
Vómitos
Irritabilidad
Anorexia
Hepatomegalia
Compromiso de conc.
Hemorragias
Esplenomegalia

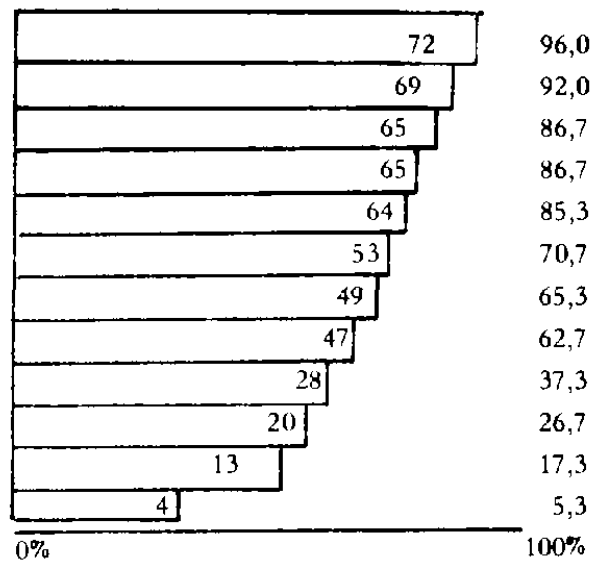

Hubo un ligero predominio de fallecidos del sexo masculino, lo que tiene la misma explicación anterior. Si merece destacarse que la to- talidad de los casos fallecidos eran desnutridos, lo que ratifica la mayor gravedad que estos procesos alcanzan en este tipo de niños.

Tabla 7

LETALIDAD SEGUN EDAD Y SEXO EN 75 LACTANTES CON SEPSIS

\begin{tabular}{|c|c|c|c|c|c|c|c|}
\hline \multirow{4}{*}{ Edad en meses } & \multirow{4}{*}{$\begin{array}{l}N^{\circ} d e \\
\text { cosos }\end{array}$} & \multicolumn{6}{|c|}{ Letalidad } \\
\hline & & \multicolumn{4}{|c|}{ Sexo } & \multirow{3}{*}{ Total } & \multirow{3}{*}{$\%$} \\
\hline & & \multicolumn{2}{|c|}{ Femenino } & \multicolumn{2}{|c|}{ Masculino } & & \\
\hline & & $N^{\infty}$ & $\%$ & $N^{\circ}$ & $\%$ & & \\
\hline $1-5$ & 39 & 2 & 2,7 & 4 & 5,2 & 6 & 7,9 \\
\hline $6-11$ & 25 & 1 & 1,3 & 3 & 4,0 & 4 & 5,3 \\
\hline $12-17$ & 8 & 2 & 2,7 & 1 & 1,3 & 3 & 4,0 \\
\hline $18-23$ & 3 & 0 & 0,0 & 0 & 0,0 & 0 & 0,0 \\
\hline Total & 75 & 5 & 6,7 & 8 & 10,5 & 13 & 17,2 \\
\hline
\end{tabular}

En la tabla 8 se relaciona la letalidad de acuerdo a su etiología. Se observa un mayor número de casos de fallecidos por sepsis a enterobacter y a pseudomonas, 5 casos en cada una. Al relacionar los fallecidos con los casos que enfermaron por una determinada etiología se establece la mayor gravedad de las sepsis por pseudomonas, ya que de 12 murieron 5 , lo que hace un $41,7 \%$. No se observó letalidad en las sepsis por Klebsiella.
Tabla 8

LETALIDAD SEGUN ETIOLOGIA EN 75 CASOS DE LACTANTES CON SEPTICEMIA

\begin{tabular}{lccc}
\hline Etiologia & $N^{0}$ casos & $N^{\circ}$ fallec. & $\%$ \\
\hline Enterobacter sp. & 34 & 5 & 6,6 \\
Pseudomonas & 12 & 5 & 6,6 \\
Klebsiella & 7 & 0 & 0,0 \\
Otros & 22 & 3 & 4,0 \\
\hline Total & 75 & 13 & 17,2 \\
\hline
\end{tabular}


Sensibilidad in vitro. En el gráfico 2 se puede apreciar el estudio en el caso del enterobacter. Seleccionamos esta etiología, ya que se presentó con la mayor frecuencia. Desgraciadamente los antibióticos que se señalan no fueron investigados en todas las cepas, lo que puede limitar las conclusiones. Así, la Gentamicina se estudió en la totalidad de los casos, alcanzando un a sensibilidad de 67,6\%. Merece destacarse la situación de la Ampicilina en que de 22 cepas el $100 \%$ resultó resistente.

GRAFICO 2

ESTUDIO DE SENSIBILIDAD IN VITRO POR SISTEMA DE DIFUSION EN PLACAS DE 34 CEPAS DE ENTEROBACTER SP.

Rifampicina
Cloramfenicol
Aminosidina
Sulfa-trimetoprim
Gentamicina
Sisomicina
Carbenicilina
Ampicilina

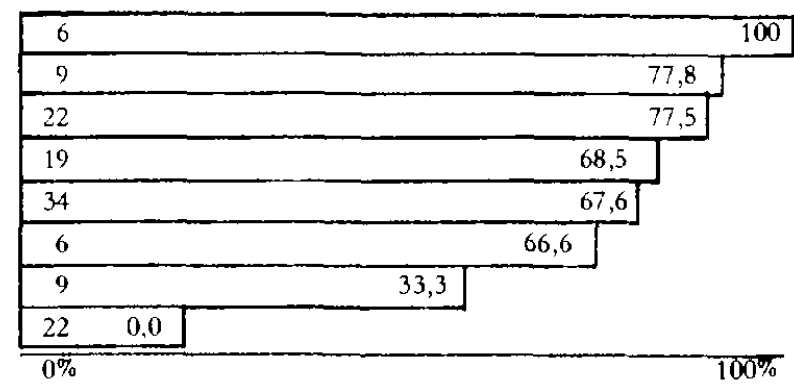

Tratamiento. El el gráfico 3 se señalan los distintos antibióticos empleados. Se ha confeccionado en frecuencia decreciente, anotando el promedio de días de uso, las dosis fueron las habituales, la vía fue de preferencia endovenosa los primeros días y luego la intramuscular. La sulfatrimetoprima se empleó vía oral.

Si consideramos que los casos estudiados fueron 75 , resulta que cada uno recibió antibióticos simultáneos o sucesivos. El criterio que se consideró fue la sensibilidad in vitro, sin embargo cuando no se observó respuesta in vivo se reemplazó por antibióticos "probables" de acuerdo al germen y que no habian sido estudiados al antibiograma.

\section{GRAFICO 3 \\ ANTIBIOTICOS UTILIZADOS EN 75 CASOS DE LACTANTES CON SEPSIS}

Gentamicina

Sulfatrimetoprima

Dicloxacilina

Aminosidina

Cloramfenicol

Carbenicilina

Sisomicina

Lincomicina

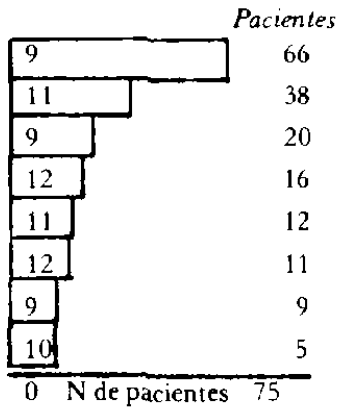

\section{COMENTARIO}

Los procesos sépticos del lactante mantienen su permanente actualidad derivada de su relativa frecuencia, dificultad diagnóstica, gravedad de los cuadros clínicos y complicaciones como shock séptico y coagulación intravascular diseminada. A esto se suma el actual predominio de gérmenes Gram (-), especialmente de los llamados patógenos oportunistas y que con frecuencia, por ser de origen hospitalario, son resistentes a los antibióticos de empleo habitual.

Si consideramos la mayor sobrevida de enfermos crónicos, graves, con déficit inmunitario, a to que se suma la creciente utilización de artefactos e instrumentos que actuán como vía potencial de contagio, es que se hace evidente la necesidad de un enfoque serio en cada Servicio. Los aspectos que parecen más importantes son:

1. Vigilancia del aseo y control de vectores.

2. Control de técnicas y de asepsia en el material empleado.

3. Adecuado lavado de manos de los médicos y personal para examen y atención de pacientes.

4. Vigilancia bacteriológica del medio.

5. Identificación de las cepas más frecuentes y su sensibilidad habitual. 
6. Utilización de antibióticos en base a antecedentes téenicos para cada etiología.

7. Implementación de laboratorios con niveles crecientes de complèjidad, desde el hospital rural al Hospital Regional.

8. Actualización periódica de conocimientos de los profesionales involucrados en el problema.

9. Derivación oportuna de pacientes de hospitales con menos recursos.

10. Recopilación y divulgación de la experiencia local.

\section{RESUMEN}

Se presenta la experiencia en sepsis del lactante durante cinco años en el Servicio de Pediatría del Hospital Regional "Leonardo Guzmán" de Antofagasta. Se analizan 75 casos de los cuales 69 tienen hemocultivos positivos. Los 6 restantes presentan cuadro clínico manifiesto de sepsis con hallazgo del germen en localizaciones secundarias. Se comprueba que el Enterobacter sp. es el agente etiológico más frecuente, aislándose en 34 casos $(45,3 \%)$, le siguen las Pseudomonas con 12 casos (16\%), KJebsiella con $7(9,3 \%)$, estafilococo coagulasa positivo con $4(5,3 \%)$, est filococo coagulasa negativo con $4(5.3 \%)$ distribuyéndose el resto entre distintos gérmenes.

Se destaca que la gran mayoría de los casos se presentan en lactantes menores de 6 meses ( $52 \%$ ), disminuyendo luego en forma progresiva, 33,3\% entre 7 y 12 meses, $10,7 \%$ entre 12 y 17 meses y sólo $4 \%$ entre 18 y 23 meses. Hubo predominio del sexo masculino con 50 casos $(67 \%$ ) y en desnutridos, con 53 casos $(70,7 \%)$. 39 casos se presentaron en el verano $(52 \%)$ y el resto se distribuyó en forma semejante en las demás estaciones del año.

E] diagnóstico de ingreso fue mayoritariamente el de Diarrea Aguda con Deshidratación, con 51 rasos (68\%), seguido de Infecciones Respiratorias Agudas en 9 casos (12\%). En sólo 5 casos $(6,6 \%)$, el diagnóstico de sepsis se planteó al ingreso.

El promedio de hospitalización fue de 30 días $y$ el rango fluctuó entre 6 y 76 días. Los hallazgos clínicos más frecuentes fueron decaimiento, 72 casos, diarrea, 69 casos, fiebre y palidez en 65 casos y deshidratación en 64 casos. La esplenomegalia sólo se encontró en 4 casos.

Se analizan los días de hospitalización, la duración de la deshidratación, fiebre, alteración de deposiciones y de mantención de hidratación parenteral

En cuanto al recuento de blancos, se presentó leucocitosis en 27 casos $(37,5 \%)$, leucopenia en $5(7 \%)$ y cifra normal en el $55.5 \%$ restante.

La letalidad fue de 13 pacientes $(17,2 \%)$ y se observó tan sólo en desnutridos. Fue más frecuente a menor edad. La etiología por pseudomonas presentó la letalidad específica más elevada con $41,7 \%$. No hubo letalidad en los casos por Klebsiella.

Referente a la sensibilidad in vitro, se analiza la estu- diada en casos por Enterobacter sp., variando desde $100 \%$ a la Rifampicina a $\mathbf{0 \%}$ a la Ampicilina.

En cuanto a los aminoglucósidos la sensibilidad fue de $77,5 \%$ para la Aminosidina, de $67,6 \%$ para la Gentamicina y de $66,6 \%$ para la Sisomicina.

En relación al tratamiento, se empleó un promedio de 2,4 antibióticos por enfermo y el criterio de uso fue la sensibilidad del antibiograma y la respuesta in vivo. El antibiólico más usado fue la Gentamicina que se empleó en 66 enfermos.

\section{SL.MMARY}

The experience on sepsis of the suckling is submitted at the Pediatrics Center of the "Leonardo Guzmán" Regional Hospital in Antofagasta during a five year period. 75 cases are analyzed out of which 69 carry positive hemocultures. The 6 remaining ones schow a manifest clinical picture of sepsis, the presence of the germ having been detected in secondary localizations. It is thereby proved that the enterobacter sp. is the most frequent aetiological agent isolating itself in 34 cases $(45,3 \%)$, being followed of the pseudomonas with 12 cases $(16 \%)$, klebsiella with $7(9,3 \%)$, positive coagulase staphylococcus with $4(5,3 \%)$, negative coagulase staphylococcus with $4(5,3 \%)$, the rest being distributed amongst different germs.

It is pointed out that the great mejority of the cases are observed in sucklings under the age of 6 months $(52 \%)$, subsequently decreasing progressivery, $33.3 \%$ between 7 and 12 months, $10.7 \%$ between 12 and 17 months and oniy $4 \%$ between 18 and 23 months. There was a majority of the masculine sex with 50 cases $-(67 \%)$ and in the underfed with 53 cases $(70,7 \%) .39$ cases turned up in summer $(52 \%)$ and the rest were distributed in a similar manner in the remaining seasonsof the year.

The diagnosis on arrival was rhiefly that of acute diarthea with dehydration. with 51 cases $(68 \%)$, followed by acute respiratory infections in 9 cases- $(12 \%)$. The sepsis diagnosis was posed on arrival in only 5 cases $(6,6 \%)$.

Ihe hospitalization average was of 30 days, the shorter period being 6 days and the congest 76 . The most frequent clinical symptoms were that of drowsiness. 72 cases, diarrehea, 69 cases, fever and paleness in 65 cases and dehydration in 64 cases. Splenomegaly was only found in 4 cases.

The hospitalization period, the duration of dehydration, fever, alteration of depositions and the period of parenteral hydration are analyzed.

In regard to the white cell count, leukocytosis was observed in 27 cases $(37,5 \%)$, leukopenia in $5(7 \%)$ and normal value in the remaining $55.5 \%$.

The death rate was of 13 patients $(17,2 \%)$, and it was observed solely in the underfed. It was more frequent as they decreased in age. The aetiology by pseudomonas showed the highest specific death rate with $41,7 \%$. There was no death rate in the klebsiella cases.

With reference to sensibility in vitro, the one studied in cases by enterobacter sp., is analyzed varying from $100 \%$ towards Riphampycin to $0 \%$ towards Ampicillin.

In regard to the aminoglucosides, the sensibility was of $77,5 \%$ towards Aminosidin, of $67,6 \%$ towards Gentamycin and of $60,6 \%$ towards Sisumycin. 
With respect to treatment, an average of 2,4 antibiotics per sick person was employed and the criterior with respect to their use was the sensibility to the antibiogram and the response in vivo. The most used antibiotic was Gentamycin which was employed on 66 patients.

\section{REFERENC:IAS}

I Blair R.; Wise, A. y Mackay, A.G. Gram negative bacteraemic shock. Mechanisins and management. J.Amer. Med. Ass. 207/2 (333-336), 1969.

2 Del Rio, L., Caballero E., Eglinton, G. y Cassorla, E. Septicemias por bacilos (iram negativos en el lactante. Revista Chilena de Pediatría, Vol. 6. (343-348), 1966.

i Feron, J.P., Saint Martin, J, Josso I,J. et al. Coagulopathies de consommation chez l'enfant. Arch. Franfaises de Ped. 28, 473, 1964

+ Mac Millan C., Weiss, A., Johnson, M. Trastornos adquiridos de la coagulación en niños. C:lin. Ped. de Norteamérica. Nov. (1029-1046), 1972

" Olcese, A. y Dal'Borgo, P. Sepsis por bacilos Gram negativos en el lactante. Rev. (hil. Ped. Vol. 9-10)(605612), 1971.

i Reyes, $H$. y Hurlado. R. Septicemias por bacilos Gram Negativos, experienca en 34 casos. Rev. Méd. Chile 92, 20, 1964

7 Puentes, $R$. y col. Sepsis a citrobacter en el lactante. Rev. Chilena Pediátrica. Vol. 46. N $\mathrm{N}^{\circ}$ (211-217), 1975.

- Luts del Río, M. y col. Septicemias por bacilos (iram negativos en el lactante. Rev. Chil. Pediatría 37:(343348), 1966.

- Corrigan, Jr., J.J. Trombocytopemia: A laboratory sign of septicemia in infants and children. J. Pediat. 85 (2): $219-221,1974$.

10 Opitz-Schmid. Enciclopedia Pediátrica. Editorial Morata. Tomo V, 452, 1967

1 Berstem, J. Brown, A.K. Sepsis and jaundice in early infancy. Pediatrics, 29, 87,3, 1962

1.2 Heffner, R., Wand Smith, G.F. Lathyma gangrenosim in pseudomonas septicemia. A.M.J. of Child. 99:524, 1960 .

1.1 Meneghello. Pediatria. $2^{\alpha}$ Edición, 1978.

14 Velson. Vaughan y McKay. Tratado de Pediatria. $6^{\alpha}$ Edicion, 1971.

15 Scroggie-Steeger. Tratado de Pediatria. 1960.

it Talbot, C.M. Septicemie due to (iram negative bacilli. Lancet. 1:668, 1962.

17 Harlam, W.R. Ciram negative sepsis, another piece of the mosaic. New Engl. J. Méd. 281: 1127, 1969.

in Cratg, W.B. Bacterial (endotoxin) shock. Méd. (ilin. N.A. $16: 404,1962$.

19) Rodriguez, F.E. Bleding due to increased intravesicular coagulation. J. Ped. 74: 163, 1969.

20 Karjsatkin, M. Diagnosis and management of disseminated, intravascular coagulation. Ped. Cilin. N.A. $18: 23,1971$.

"1 Hoffmamn, M.A. and Finberg, L. Pseudomona infection in infants assoriated with high humidity environment. J. Pediat 46:626, 1955.
22 Prerce, A.K. et als. An analysis of factors predisposing to gram negativy bacillary necrotizing pneumonia. Am. Rev. Resp. Dis. 94:309, 1966.

23 Finland, M., Jones Wand Bames, M. Ocurrence of seruis bacterial infection since introduction of antibacterial agents. J.A.M.A. 170:2188, 1959

2t Jhonstons, B., Sel, S.H. Septicemie in infants and childrens. Pedial 34:473, 1964.

25 Redman, Lockey. Colonization of the upper respiratory tract with Gram negative bacilli after operations, endotiacheal intubation and prifilactic antibiotic therapy. Anaesthesia, 22:2. 1967.

26 Keller et. al. Septicemic candidrass in infants: a case presentation and literature review. Am. J. I) Child. 131 (11): 1260-3, Nov. 1977

27 Falla, A. et al. Candida sepsis and disseminated intravascular coagulation in postoperative infants. Cilin. Pediatr. (Phila) 14(3):294-6, marzo 75

2k Pieraet, $C$. et al. Salmonella Wien epidemic in pediatric word. Pediatric 29(6): 595-606, 1974

2.) Watt, P.J., Okubadejo, O.A. Changes in incidence and actionology of bacteremic in hospital practice. Brit. Med. J. 1:210, 1967

3" Mc Cobe, W.R. and Jackson, G.G. Gram negative bacteremie. Arch. In. Med. 110:847, 1962.

31 Senra del Valle D., Imbrogno, M. y Fernández, E. Uso de la gentamicina en Pediatria en Infecciones producidas por Gram negativos. Symposium latinoamericano sobre infecciones y gentamicina. Marzo 10-11. Rio de Janeiro, Brasil, 1969.

12 Jorge Jiménez de la Jara, Istdiro Horwitz y Enrique Fanta. Infección severa por Pseudomonas aeuroginosas. Irat. con carbenicilina. Pediatria 15:263-266, 1972.

3s Isabel, K.G. et al. Treatment of Meningitis and septicemie in infancy with sulphamethoxazole/timethoprim combination. Acta Paediatr. Scand. 64(1) 25-32. Jan 75 .

34 Chalmers, J.P., Taller, D.J. Effects of trealment on the mortality rate in septicemie. But. Med. J. 2:338, 1969.

15. Gross, $S$. and Methorm, D. Excharge transfusion for intravascular coagulation. J. Pediat. 78:415. 1971.

3i Barson et als. Fatal pseudomona aeuroginosa bronchopneumonia in a children's hospital. Arch. I is. of childr. 46:55, 1971.

37 Asay et als Pseudomonas infection in infants and rhildrens. New Eng. Jour. Med. 262:1062, 1960.

3* Howard, J. y alumnos. Bolecín Ministerio de Salud de Chile. Vigilancia de enfermedades transmisibles y zoonosis. Vol $\mathrm{r}, \mathrm{N}^{0} 12(3-5)$. Dic. 1978

3:1 Jones. R.J. et als. Profilaxis and therapy for pseudomonas infections with carbenicillin and gentamicin. Brit. Med. Jour. 3:79, 1967

"t Gellts and Kagan. Current Pediatric Therapy, 1978

$\checkmark$ Krugman y Ward. Enfermedades infecciosas. Quinta edición, 1974

$\because$ Goodgman y Gilmon. Bases Farmacológicas de la Terapéutica Cuarta Edición, 1974.

4.s Armijo. Curso de Epidemiología. 1964. 
it Opitz y Schmid. Enciclopedia Pediátrica. Tomo III. 1967.

t5 McHenry: Bacteremic due to Gram negative bacilli. Am. Int. Med. 56:207, 1962. 4h Katz, Sol. Gram negative bacteremic. Med. Clin. N A. $46: 482,1962$.

47 Burrows. Tratado de Microbiología. $19^{a}$ Edición 1969. 